This item was submitted to Loughborough's Research Repository by the author.

Items in Figshare are protected by copyright, with all rights reserved, unless otherwise indicated.

\title{
Dynamic analysis of wind power integration into the Northern Interconnected Power System of Chile
}

PLEASE CITE THE PUBLISHED VERSION

http://dx.doi.org/10.1109/chilecon.2015.7400446

PUBLISHER

(C) IEEE

VERSION

AM (Accepted Manuscript)

\section{PUBLISHER STATEMENT}

This work is made available according to the conditions of the Creative Commons Attribution-NonCommercialNoDerivatives 4.0 International (CC BY-NC-ND 4.0) licence. Full details of this licence are available at: https://creativecommons.org/licenses/by-nc-nd/4.0/

\section{LICENCE}

CC BY-NC-ND 4.0

\section{REPOSITORY RECORD}

Vallejos, Mauricio, Marcelo Cortes-Carmona, Francisco M. Gonzalez-Longatt, and Edward Fuentealba. 2019. "Dynamic Analysis of Wind Power Integration into the Northern Interconnected Power System of Chile". figshare. https://hdl.handle.net/2134/21532. 


\title{
Dynamic Analysis of Wind power integration into the Northern Interconnected Power System of Chile
}

\author{
Mauricio Vallejos, Marcelo Cortés-Carmona, Member, IEEE, Francisco González-Longatt, Edward
} Fuentealba

\begin{abstract}
Energy policy of Chile establishes that by 2025, at least $20 \%$ of the annually generated energy must come from renewable energy sources. In this context, many projects of solar and wind farms have been presented for connection in ten Chilean electrical systems. The aim of this study is to evaluate the transient behavior of the Northern Interconnected System (SING) regarding the high levels of integration of wind generation. The behavior of the most important wind farms under construction and / or approved for construction is analyzed. A comparative study of the technologies of fixed speed, doubly fed and full converter is carried out in two points of the system employing software for electrical system analysis, DigSILENT. The main results show that technologies of fixed speed and doubly fed have a better response in the voltage and reactive power variables, meanwhile the angle and reactive power performs better in a full converter technology.
\end{abstract}

Index Terms — Energy conversion, Power system dynamics, Power system simulation, Wind energy integration,

\section{INTRODUCCIÓN}

La generación de electricidad mediante energías renovables se expande velozmente, y se espera que supere al gas natural y que doble a la energía nuclear hacia el año 2016, convirtiéndose en la segunda fuente más importante a nivel mundial, después del carbón [1]. Se estima que al año 2018 la generación de electricidad mediante energías renovables, a nivel global, alcanzará un $25 \%$ de la generación bruta.

La capacidad de generación de electricidad mediante energía eólica en todo el mundo alcanzó, el año 2012, los 282.275 [MW], de los cuales 44.609 [MW] fueron instalados en el 2012 [2].

De estos 44.609 [MW] instalados el 2012, Asia, representó la mayor parte de las nuevas instalaciones $(36,3 \%)$, seguida de América del Norte $(31,3 \%)$ y Europa $(27,5 \%)$, América Latina un 3,9\% y Australia / Oceanía un 0,8\%, África por su

This work was supported in part by Education Ministry of Chile Grant PMI ANT 1201 as well as CONICYT/FONDAP/15110019 "Solar Energy Research Center" SERC-Chile.

Marcelo Cortés-Carmona, Mauricio Vallejos, y Edward Fuentealba, están con la Universidad de Antofagasta, Facultad de Ingeniería, Departamento de Ingeniería Eléctrica, Antofagasta, Chile, Tlf. +56-55-2637475, E-mail: marcelo.cortes@uantof.cl, edward.fuentealba@uantof.cl,

Francisco González-Longatt está con Loughborouh University, School of Electronic, Electrical and Systems Engineering W2.63, Loughborough, LE11 3TU, United Kingdom +44(0)150 9227061, E-mail: fglongatt@,fglongatt.org. parte, sólo contribuyó en un $(0,2 \%)$ y sigue siendo un mercado pequeño para la energía eólica.

La Asociación Mundial de Energía Eólica (WWEA, World Wind Energy Association) espera una capacidad global de más de 500.000 [MW] para el año 2016 y alrededor de 1.000.000 [MW] hacia el año 2020.

El año 2012 América Latina y Europa del Este fueron las regiones del mundo con las mayores tasas de crecimiento, donde cuatro países de América Latina mostraron un alto crecimiento: Argentina con un $80 \%$, Brasil con $75 \%$, Nicaragua con $62 \%$ y México con un crecimiento del $45 \%$ mostraron los mayores crecimientos en la región.

Chile presentó en el año 2014 una producción bruta de energía eléctrica de 69.897 [GWh], de los cuales 1.411 [GWh] $(2.0 \%)$ fueron generados mediante el uso de energía eólica [3].

El presente trabajo tiene por objeto evaluar la respuesta del Sistema Interconectado del Norte Grande (SING) ante la integración de parques eólicos para diferentes tecnologías, tamaños y ubicaciones. El artículo se ha organizado de la siguiente manera; En la sección II se presentan las tecnologías utilizaras para producir energía eléctrica a partir del viento, En la sección III se valida el modelo de generador que se utilizará con la tecnología convertidor completo. En la sección IV se describe el SING y los proyectos eólicos que se analizarán, En la sección $\mathrm{V}$ se presentan los resultados de los casos analizados, y finalmente en la sección VI se entregan las conclusiones y sugerencias para futuros trabajos.

\section{TECNOLOGÍAS UTILIZADAS EN TURBINAS EÓLICAS}

\section{A. Generadores eólicos de velocidad fija}

La tecnología eólica más simple que se utiliza para producción de energía es la denominada de velocidad fija (FSWT, Fixed speed wind turbine). El convertidor FSWT, está compuesto por un generador de inducción, el cual gira a velocidad fija gracias a una caja de engranajes que transforma la velocidad de giro de las aspas a la velocidad necesaria en el rotor para generar energía a la frecuencia de la red, e inyectarla directamente a esta. La Fig. 1, muestra un diagrama de esta turbina.

Como es sabido, la operación de una máquina de inducción como generador demanda potencia reactiva, la cual debe suministrarse mediante bancos de condensadores, o desde la misma red. Adicionalmente, este tipo de convertidores requiere una compensación de reactivos para enfrentar las 
variaciones de tensión en el Punto de Conexión (PCC), la cual generalmente alcanza un $30 \%$ de la capacidad del parque [4].

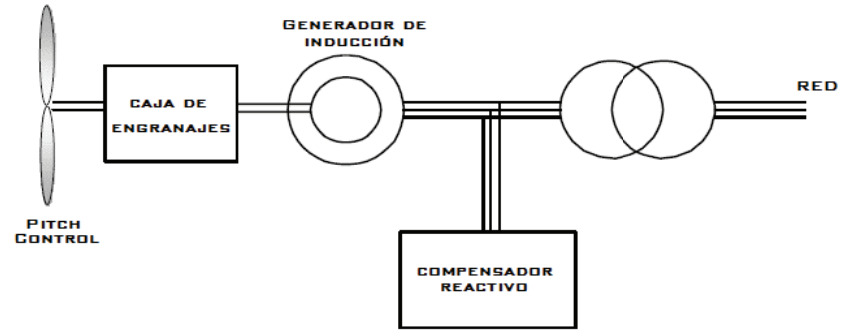

Fig. 1: Estructura general de un convertidor FSWT.

Este tipo de sistemas presenta limitaciones debido a que para su operación requiere velocidades de viento dentro de un rango limitado, y no puede inyectar potencia reactiva para caídas de tensión en el PCC.

\section{B. Generadores é́licos doblemente alimentados}

Los convertidores de velocidad variable doblemente alimentados (DFIG, Double Feeded Induction Generator), como el que se muestra en la Fig. 2, utilizan un generador de inducción de rotor bobinado, cuyos devanados están conectados, en el caso del estator, directamente a la red, mientras que el rotor se conecta a la red mediante un convertidor "back-to-back" basado en IGBTS's. El convertidor del lado del rotor regula las potencias activa y reactiva inyectadas a la red, mientras que el convertidor conectado a la red controla la tensión en la barra de corriente continua.

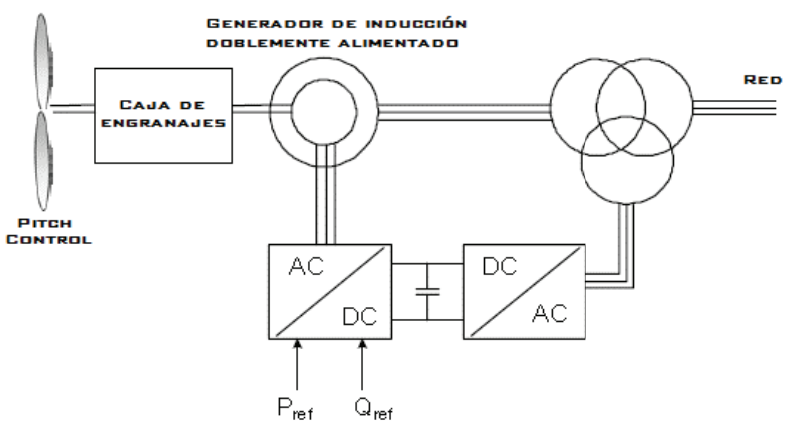

Fig. 2 : Estructura general de un convertidor DFIG.

\section{Generadores eólicos con convertidor completo}

Por su parte, la tecnología de convertidores completos (FCWT, Full Converter Wind Turbine), se puede utilizar ya sea con: generadores asincrónicos o sincrónicos de imanes permanentes en los modelos con caja de engranajes y/o con generadores sincrónicos de polos salientes (GSPS) o generadores sincrónicos de imanes permanentes (GSIP) para los modelos sin caja de engranajes [5]. El generador de imanes permanentes presenta una serie de ventajas técnicas en términos de mantenimiento, tamaño y reducción de costos al no necesitar engranajes, razones por las cuales es de interés considerar este tipo de generadores en el estudio de la tecnología FCWT.

La principal ventaja del convertidor FCTW radica en el desacoplo entre la red y el generador, gracias a los convertidores conectados en cascada, lo cual permite minimizar los efectos de una falla en la red y su propagación al generador, y por lo tanto, proporciona una mejor respuesta ante fallas [6]. En general, un generador eólico FCWT tiene una configuración como se muestra en la Fig. 3.

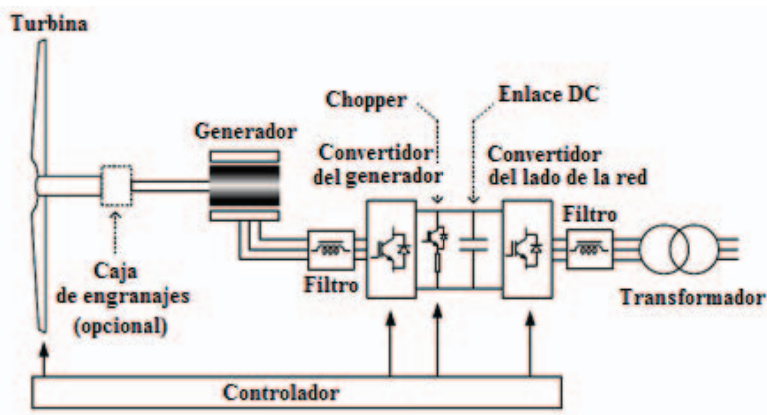

Fig. 3 : Estructura general de un convertidor FCWT.

La función del convertidor conectado al generador es la de regular la velocidad y potencia del generador. Alternativamente este convertidor puede ser utilizado para controlar la tensión en el enlace DC.

La estrategia de control de este convertidor dependerá del tipo de generador utilizado y sus características. Este generalmente se realiza mediante técnicas de modulación vectorial SVM (Space Vector Modulation), en las cuales el eje directo está alineado con el flujo rotacional del estator.

El control de las potencias activa y reactiva se realiza a través del convertidor del lado de la red, el cual proporciona una mayor flexibilidad de proveer potencia activa a la red sobre todo durante fallas. El relativo desacople existente entre el control de potencias activa y reactiva da paso a distintas estrategias de control con cierto grado de independencia [6].

\section{MODELOS DE GENERADOR PARA UN CONVERTIDOR COMPLETO}

Una de las principales características de la tecnología FCWT son los convertidores en cascada que desacoplan al generador de la red, lo cual protege al generador de cualquier evento producido en ésta. Para este estudio se busca analizar el comportamiento de la tecnología FCWT con GSIP. Actualmente el software de modelamiento DigSILENT no cuenta con un modelo específico para este tipo de generadores, sin embargo, algunos autores [7], [8] han concebido métodos de control de la excitación, AVR (Automatic Voltage Regulator), de manera de emular el comportamiento de un GSIP en el software [8].

Considerando este hecho, se realizaron simulaciones en el SING utilizando el modelo de control de la corriente excitación propuesto en [8], [9]. El sistema SING, posee un gran número de elementos y controladores, por lo que para lograr la convergencia se requirió utilizar pazos de integración muy pequeños, lo cual genera tiempos de simulación 
demasiado elevados e inestabilidad en algunos controladores de la base de datos existente.

Una forma de superar el problema definido en el párrafo anterior es considerar lo propuesto por Achilles [7]. Este investigador prueba en sus estudios que existe una relativa equivalencia entre las componentes que se encuentran aguas arriba del inversor y una fuente de corriente, por lo que concluye que la naturaleza del generador no implica una diferencia para las variables eléctricas vistas desde el lado de la red ante la ocurrencia de una perturbación aguas debajo de los terminales del inversor.

Para corroborar esto, se realizó la comparación de la respuesta de ambos tipos de control. En primer lugar se simula un GSPS con un AVR genérico, y en segundo lugar se emula un GSIP mediante la utilización de un GSPS excitado mediante un AVR que mantiene la corriente de excitación constante.

Se modelaron dos parques eólicos conectados a una red externa representada por una fuente de tensión. Cada parque consta de 30 aerogeneradores de 1,5 MVA cada uno, completando un total de 45 MVA. El parque $\mathrm{N}^{\circ} 1$ emula el generador sincrónico de imanes permanentes. El parque $\mathrm{N}^{\circ} 2$ está modelado con un generador sincrónico de polos salientes. Se simula una falla correspondiente a un cortocircuito trifásico en el punto de conexión de ambos parques en $\mathrm{t}=0[\mathrm{~s}]$, la cual es despejada luego de 150 [ms].

Tal como se indica en [9], la diferencia en los modelos de las excitaciones de ambas máquinas, no se ve reflejada en el comportamiento de las variables eléctricas de salida del inversor hacia la red, (Potencia activa y reactiva, voltajes, velocidad y ángulos). En efecto, la Fig. 4 muestra que para ambos modelos las variables potencia activa, potencia reactiva y tensión tienen exactamente la misma respuesta para ambos modelos.

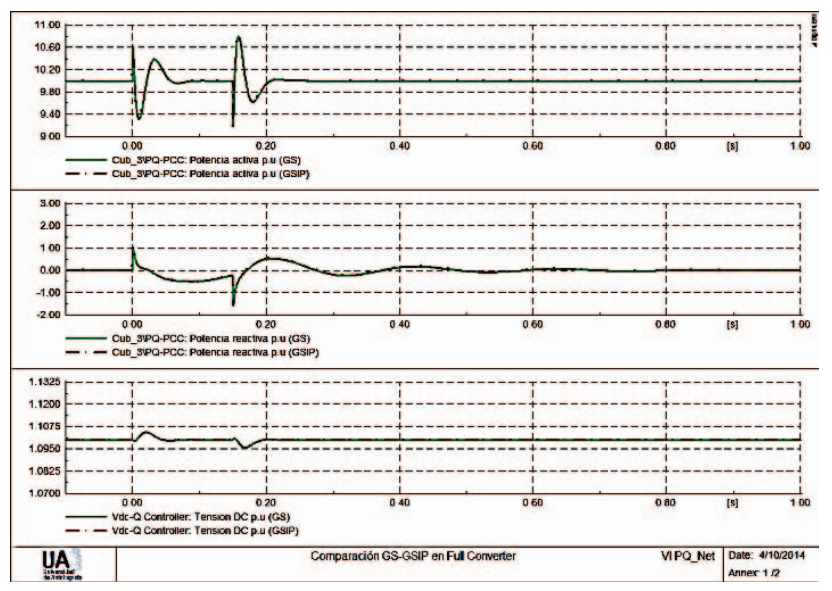

Fig. 4: Respuesta de las variables eléctricas en el lado de la red.

Bajo el enfoque de este estudio, el cual se centra en el comportamiento e interacción del convertidor eólico con la red ante perturbaciones en el PCC, y en vista de los resultados observados en este apartado, se considerarán equivalentes las respuestas tanto en parques modelados con GSPS como para aquellos con GSIP en generadores del tipo FCWT. Por lo tanto, las simulaciones para el convertidor FCWT se realizarán con GSPS.

\section{INTEGRACIÓN DE GENERACIÓN EÓLICA EN EL SING}

El Sistema Interconectado del Norte Grande, abastece la zona norte de Chile, desde Arica y Parinacota por el norte hasta la localidad de Coloso en el límite sur de Antofagasta. Este sistema representa aproximadamente el $27 \%$ de la capacidad instalada total en el país, atendiendo el $7,5 \%$ de la población nacional. Su generación es principalmente orientada a satisfacer el consumo de la industria minera.

En la matriz de generación del SING la principal fuente de energía es de origen térmica y utiliza como energía primaria: Carbón, Gas Natural Licuado (GNL), Diesel y Fuel Oil. La capacidad instalada del sistema para el año 2013 era de 4.607,72 MW. Por otra parte, se tiene que la generación bruta del 2013 fue 17,236 TWh/año, de los cuales 1,355 TWh/año fueron empleados en consumos propios y $0,468 \mathrm{TWh} / \mathrm{años}$ se consumieron en pérdidas de transmisión. Así, la generación neta entregada en barra a los consumidores fue 15,414 TWh/año [9]. El mismo año 2013 se tiene que la generación bruta máxima fue 2,226 $\mathrm{GW}$, mientras que la demanda máxima fue 2,060 GW. Por lo tanto, el Factor de Carga de la demanda es $85,4 \%$.

El mayor volumen de energía del SING es consumido por las grandes compañías mineras, principales clientes industriales del sistema, esta característica junto al elevado factor de carga, hacen del SING un sistema muy particular, debido a que habitualmente los sistemas eléctricos tienen un factor de carga que ronda el $60 \%$, con una alta participación de clientes regulados $(60 \%)$, como es el caso del Sistema Interconectado Central, el cual abastece la zona comprendida entre Taltal por el norte y la isla grande de Chiloé por el sur.

A partir de la promulgación de la Ley $\mathrm{N}^{\circ} 20.257$ [10] el año 2009 , la cual obliga a las empresas generadoras dependientes de combustibles fósiles, a cumplir con el requisito de que al menos un $10 \%$ de su producción debe provenir de Energía Renovable No Convencional (ERNC), se produjo que en Chile, entre los años 2009 y 2012 se han presentado para evaluación ambiental cerca de 50 proyectos de generación eléctrica mediante energía eólica. Esta ley fue posteriormente modificada mediante la reciente Ley $\mathrm{N}^{\circ} 20.698$ [11], la cual modifica la meta de un $10 \%$ de producción mediante ERNC a un $20 \%$ al año 2025.

De este modo, en el SING se han presentado para evaluación ambiental, desde el año 2009 a la fecha, los nueve proyectos eólicos que se señalan en la Tabla 1 . Al inicio de este estudio el parque eólico Valle de los Vientos se encontraba en etapa de construcción, para, finalmente entrar en operación comercial el 21 de marzo de 2014.

Basándose en la información entregada por las empresas propietarias de los proyectos, se aprecia que en la zona de Calama se concentra gran cantidad de proyectos, los cuales tendrían un punto de conexión al SING en la S/E Calama. En este escenario, resulta interesante analizar el comportamiento de las tecnologías dominantes en generación eólica al concentrarse un alto nivel de este tipo de plantas en un punto común del sistema. 
TABLA 1: PROYECTOS EÓLICOS PRESENTADOS PARA EVALUACIÓN AMBIENTAL EN EL SING.

\begin{tabular}{|c|c|c|c|}
\hline Proyecto & $\begin{array}{c}\text { Fecha de } \\
\text { presentación }\end{array}$ & $\begin{array}{c}\text { Potencia } \\
\text { instalada } \\
{[\mathrm{MW}]} \\
\end{array}$ & Estado \\
\hline Parque eólico Sierra Gorda Este & $20-12-2013$ & 168 & En calificación \\
\hline Parque eólico Tchamma & $18-12-2013$ & 273 & En calificación \\
\hline Parque é́lico Calama B & $10-09-2012$ & 75 & Aprobado \\
\hline Parque eólico Andes Wind Park & 24-07-2012 & 65 & Aprobado \\
\hline Parque eólico Calama A & 22-06-2012 & 108 & Aprobado \\
\hline Parque eólico Loa & $30-05-2012$ & 528 & Aprobado \\
\hline Parque eólico Calama & 07-06-2011 & 128 & Aprobado \\
\hline Parque eólico Ckani & 04-05-2011 & 240 & Aprobado \\
\hline Parque eólico Valle de los vientos & $16-04-2009$ & 99 & Aprobado \\
\hline
\end{tabular}

El proyecto parque eólico "Valle de los vientos" se compone de 45 turbinas de entre 2,1 y 2,3 MW cada una, teniendo un factor de planta esperado de $31 \%$. Se proyectó en un principio que el Parque Eólico entre en servicio a mediados del año 2012, sin embargo, su operación comercial se inició el 27 de marzo de 2014.

El punto de conexión contemplado para el Parque Eólico es la barra existente de $110 \mathrm{kV}$ de la S/E Calama, a través de una línea de transmisión de aproximadamente 13,2 km que une la mencionada barra con la subestación elevadora de Valle de los Vientos.

El Parque eólico Calama se proyecta con una capacidad instalada de 128 [MW]. La conexión de este parque se realizaría en la barra de $110 \mathrm{kV}$ de la sub estación Calama, mediante una línea de transmisión de 28,5 [Km]. La Fig. 5 muestra el esquema de conexión de ambos parques a la S/E Calama.

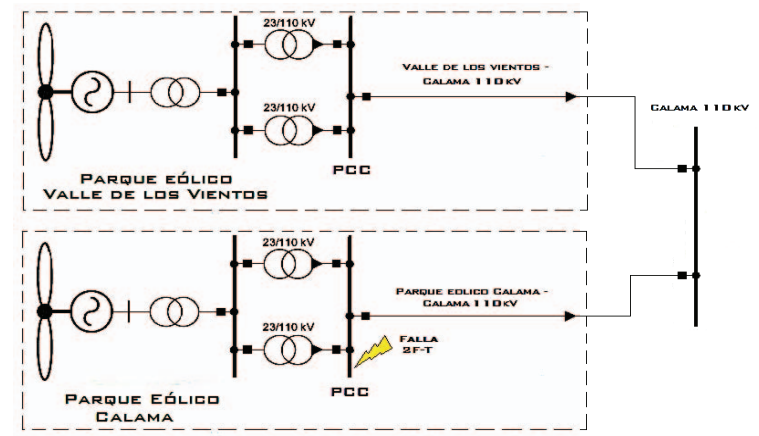

Fig. 5: Esquema de conexión parques eólicos Valle de los Vientos y Calama.

El parque eólico Loa I se ubica entre las comunas de María Elena y Tocopilla, consta de dos etapas idénticas denominadas Loa I y Loa II, ambas de 264 MW. Para la conexión de este parque se supuso una conexión directa a la ampliación de la Línea Crucero - Lagunas $220 \mathrm{kV}$, proyectada por la dirección de obras del CDEC, mediante una subestación denominada "Quillagua".

La conexión de cualquier unidad generadora al SING, está sujeta a los requerimientos técnicos impuestos por la Comisión Nacional de Energía (CNE), mediante la Norma Técnica de Seguridad y Calidad de Servicio (NTSyCS) [12].

En lo referente a parques eólicos, y la respuesta de los niveles de tensión ante fallas producidas en la red de transmisión, la normativa señala específicamente que, las unidades de un parque eólico deberán ser diseñadas de modo de asegurar que el parque se mantenga en servicio cuando la tensión en el PCC varíe, a consecuencia de una falla en el sistema, dentro de la zona achurada de la Fig. 6.

Adicionalmente, se deberá verificar que, operando el parque a plena carga y ante la ocurrencia de un cortocircuito bifásico a tierra en uno de los circuitos del Sistema de Transmisión al cual se conecta el parque, la absorción neta de potencia reactiva deberá ser nula en tanto la tensión en el punto de conexión se mantenga bajo los 0.9 pu, a excepción del período comprendido entre el tiempo máximo de despeje de la falla (TMDF) y los $30 \mathrm{~ms}$ posteriores a éste, periodo en el cual la absorción neta de potencia reactiva del parque no deberá superar el $30 \%$ de la potencia nominal de éste.

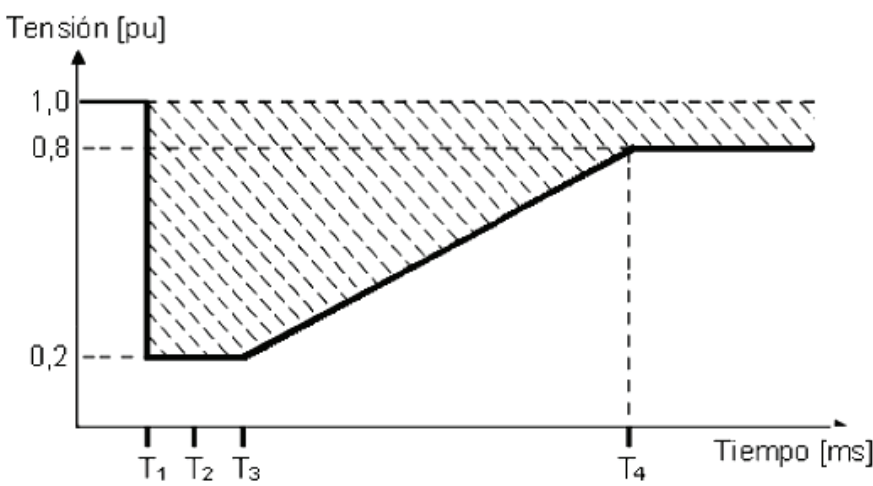

Fig. 6: Requerimientos de tensión ante fallas según NTSyCS.

Donde:

$\mathrm{T} 1=0[\mathrm{~ms}]$, Tiempo de inicio de la falla.

$\mathrm{T} 2=\mathrm{TMDF}^{1}$

$\mathrm{T} 3=\mathrm{T} 2+20[\mathrm{~ms}]$

$\mathrm{T} 4=1000[\mathrm{~ms}]$

Los requerimientos para generadores eólicos en Chile, en general, son una adaptación de la normativa alemana, y esta, en general, no dista significativamente de los requerimientos técnicos impuestos en otros países con altos niveles de generación eólica, en los que se busca proveer a las centrales eólicas con las capacidades de control y regulación existentes en las plantas convencionales, de modo de asegurar una operación segura, confiabilidad y económica de los sistemas de potencia [13].

\section{RESUltados}

Se evaluó el efecto en el comportamiento dinámico en el PCC de los parques eólicos señalados en la sección IV. El análisis del comportamiento dinámico del sistema se realiza mediante simulaciones en el software DigSILENT Power Factory. Estas simulaciones se realizaron escalando los modelos de los respectivos convertidores eólicos para alcanzar la potencia del parque completo, debido a que este estudio se centra en la interacción entre el parque y la red, y no en los efectos al interior del parque, los resultados del escalamiento no incide en mayores errores entre los modelos [14].

En cada uno de los casos analizados se analiza el comportamiento dinámico de los parques eólicos ante un 
cortocircuito bifásico a tierra producido en $\mathrm{t}=1$ [s], con una reactancia a tierra de $50[\mathrm{Ohm}]$ y un tiempo de despeje de 100 [ms] en el PCC.

\section{A. Parque eólico Loa}

El proyecto Loa es el de mayor tamaño proyectado para el SING. El parque está programado para construirse en dos etapas $256 \mathrm{MW}$ cada una. En este trabajo se simula una perturbación conforme a lo definido en el párrafo anterior para la primera etapa, es decir, un parque de $256 \mathrm{MW}$. La Fig. 7 muestra la respuesta de la potencia activa para las tres tecnologías. En estas se observa que el tiempo de recuperación para la tecnología FCWT en menor que las otras tecnologías.
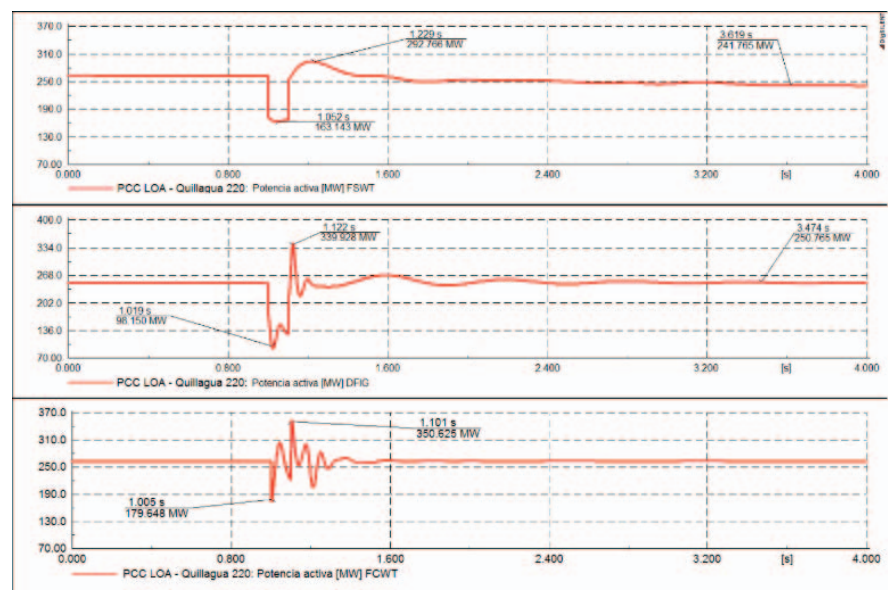

Fig. 7: Potencia activa inyectada a la red en parque eólico Loa I.

Una situación similar ocurre en la respuesta de la frecuencia que se aprecia en la Fig. 8, donde al modelar los parques mediante FSWT las oscilaciones se presentan de forma más prolongada, con DFIG, las oscilaciones tienen una menor duración, pero valores peak más pronunciados, mientras que al modelar los parques con FCWT las fluctuaciones en la frecuencia son más acotadas y de menor intensidad.

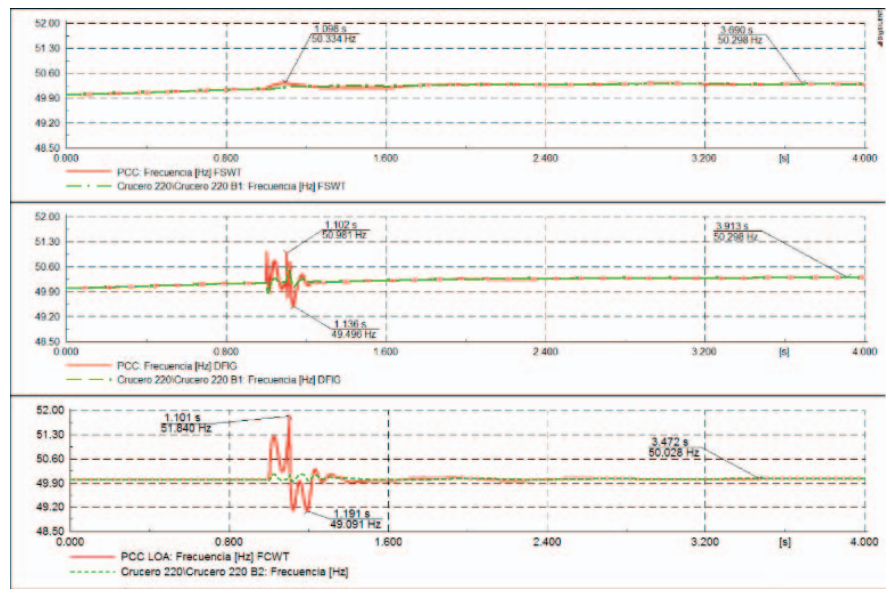

Fig. 8: Frecuencia en la red en parque eólico Loa I.

Por otra parte, la tensión se recupera para los tres casos dentro de los tiempos establecidos por las norma chilena y por normas internacionales, estos tiempos de recuperación varían según el tipo de tecnología, donde se aprecia que el parque modelado con generador de inducción doblemente alimentado tiene una respuesta mucho mejor respecto a las otras tecnologías, esto principalmente, a su capacidad de control de la inyección de potencia reactiva, en lo cual el generador FCWT se encuentra en desventaja, tal como se refleja en la Fig. 9.

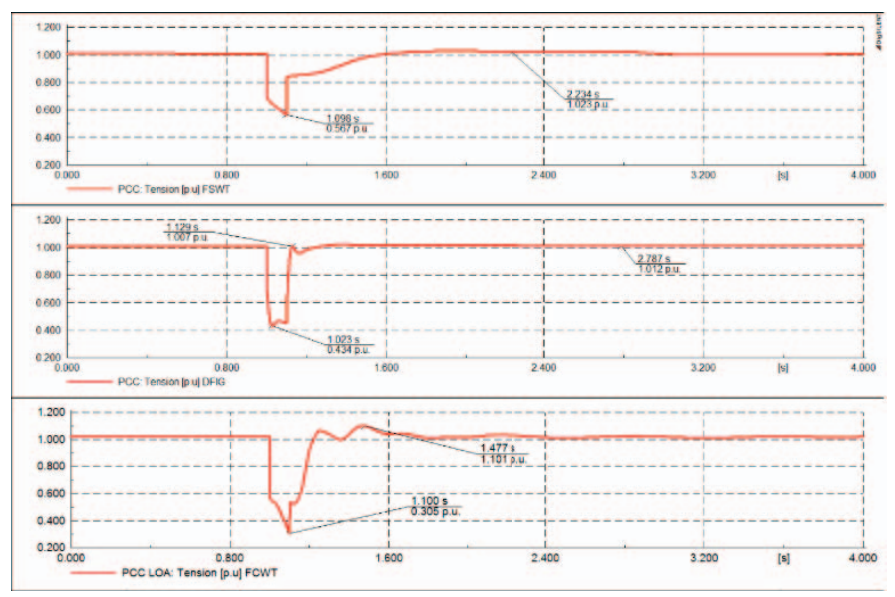

Fig. 9: Frecuencia en la red en parque eólico Loa I.

\section{B. Análisis conjunto de parque Valle de los Vientos y Calama}

En este caso se analiza una falla para el caso en que operan en barras cercanas en Calama los proyectos Valle de los vientos y Calama. En este contexto, los análisis se centrarán en el comportamiento dinámico del parque eólico, donde se verificará el cumplimiento de los requerimientos normativos especificados en la NTSyCS [12].

En la Fig. 10 se muestra el comportamiento dinámico de las tensiones en los puntos de conexión de cada parque al sistema interconectado para los tres tipos de tecnologías evaluadas en este estudio, donde, desde arriba hacia abajo, se muestran las respuestas de los parques modelados con FSWT, DFIG y FCWT.

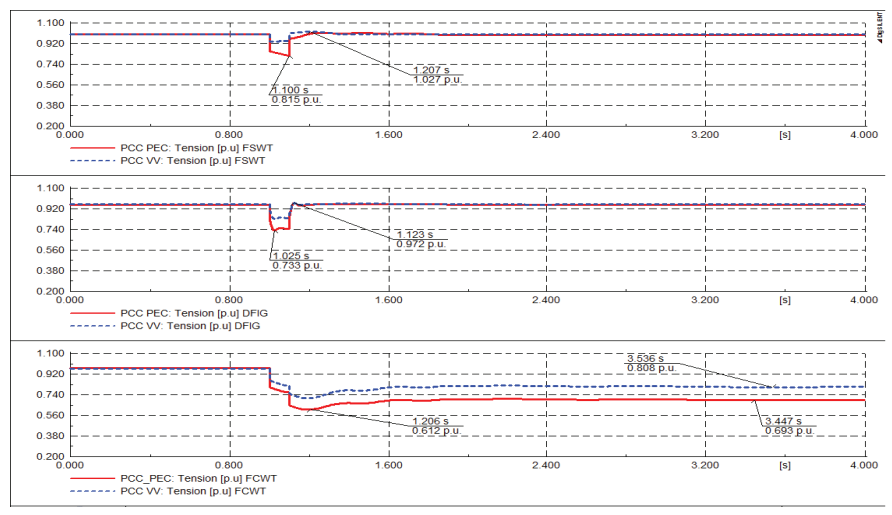

Fig. 10: Respuesta de la tensión en los PCC proyectos Valle de los Vientos y Calama.

Las simulaciones revelan que las tensiones en ambos parques se recuperan dentro de los tiempos establecidos por la normativa para los casos en que el parque esta modelado mediante las tecnologías FSWT y DFIG, estos tiempos de 
recuperación varían levemente según el tipo de tecnología. Por su parte el FCWT no es capaz de recuperar los niveles nominales de tensión, siendo, según los requerimientos de la norma chilena, necesaria la desconexión del parque que presenta la falla, en este caso, el parque eólico Calama. Esto se aprecia en la Fig. 10, donde la gráfica inferior, presenta niveles de tensión para el Parque Eólico Calama (PEC) bajo los 0.7 [p.u], mientras que el parque eólico Valle de los Vientos presenta niveles de tensión en el punto de conexión en el límite permitido por la norma, siendo específicamente 0.808 [p.u].

En lo que corresponde a la potencia reactiva inyectada hacia la red se aprecia que durante el período que se sostiene la falla, para el caso de un modelamiento mediante FSWT, el parque Valle de los vientos absorbe potencia reactiva, potencia la cual supera levemente los límites establecidos por la normativa. Por su parte al modelar el parque mediante FCWT, el parque Valle de los vientos retoma la operación normal, en términos de potencia reactiva, mientras que el parque Calama no puede recuperar la potencia nominal. La Fig. 11 presenta los resultados de las simulaciones para la potencia reactiva.

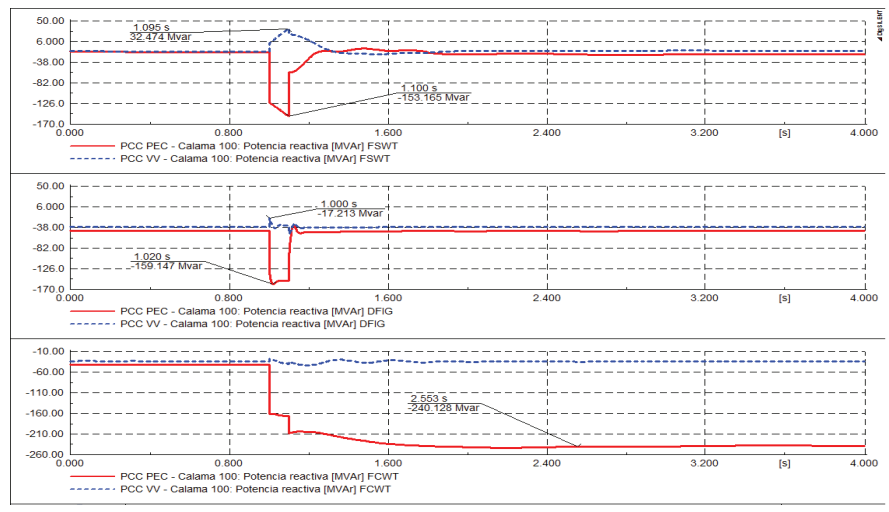

Fig. 11: Respuesta de la potencia reactiva en los PCC proyectos Valle de los Vientos y Calama.

La Fig. 12 muestra las respuestas en términos de potencia activa inyectada a la red, En esta figuras se aprecia un comportamiento estable en el parque modelado mediante FCWT, mientras que el parque DFIG presenta leves oscilaciones tras el despeje de la falla, aunque dentro de los rangos admitidos por la normativa.

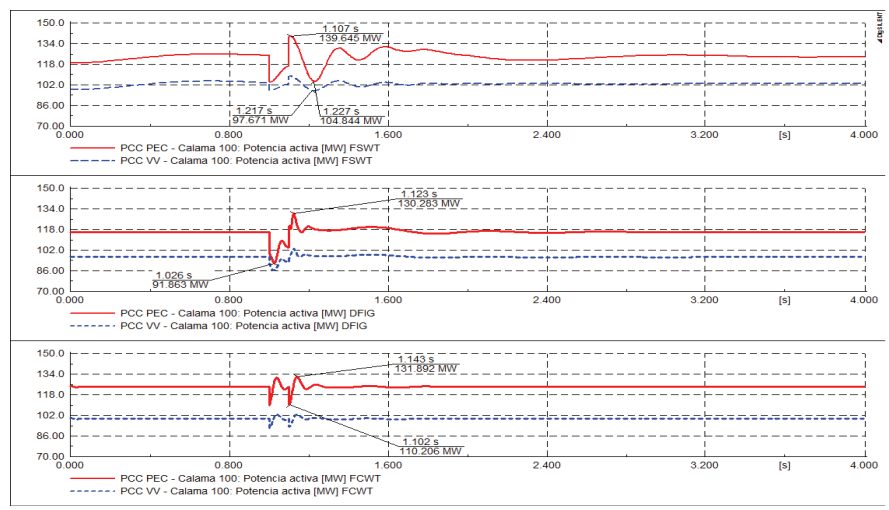

Fig. 12: Respuesta de la potencia activa en los PCC proyectos Valle de los Vientos y Calama.
Al modelar los parques mediante la tecnología de velocidad fija, estos presentan mayores dificultades en recuperar la potencia de consigna, aunque siempre dentro de los límites permitidos por la norma, en ambos parques.

\section{CONCLUSIONES}

En este estudio se ha analizado el comportamiento dinámico del SING ante la presencia de parques eólicos de distinta tamaño y con distintos puntos de conexión De estos análisis se ha podido concluir que la tecnología FCWT presenta una mejor respuesta en la recuperación de los niveles de frecuencia y potencia activa inyectada a la red. Por otra parte, la tecnología DFIG tiene en general una mejor respuesta en voltaje y potencia reactiva. Esta mejor respuesta se manifiesta en un menor tiempo de oscilación. Para el caso en que dos parques se conectan a la misma barra, la tecnología FCWT presenta deficiencias al no poder mantener la tensión en el PCC dentro de los márgenes establecidos por la normativa, por lo cual debe salir de operación según lo establece la norma técnica. Futuras investigaciones en este campo podrían incorporar el estudio de la compensación de reactivos para parques del tipo FCWT, así como los controladores asociados a esta tecnología, de modo de desarrollar una estrategia de control adecuada para casos en que más de un parque se conecte a un mismo punto de un sistema de transmisión.

\section{AGRADECIMIENTOS}

Los autores agradecen el apoyo financiero provistos por los proyectos PMI ANT 1201 del Ministerio de Educación, como así mismo del proyecto CONICYT/ FONDAP/ 15110019 "Solar Energy Research Center" SERC-Chile.

\section{REFERENCIAS}

[1] International Energy Agency «http://www.iea.org/topics/renewables/,» $23 \quad 09$ 2014. [En línea]. Available: http://www.iea.org/topics/renewables/. [Último acceso: 2309 2014].

[2] World Wind Energy Association, World Energy Report, Bonn, 2012.

[3] Comisión Nacional de Energía, «Balance Nacional de Energía 2014,» Santiago, 2015.

[4] C. Rahmann, H. Haubrich, A. Moser, R. Palma-Behnke, L. Vargas y M. Salles, «Justified Fault-Ride-Through Requirements for Wind Turbines in Power Systems,» IEEE Trans. Power Syst., vol. 26, nº 3, pp. 15551563, Aug. 2011.

[5] C. Z. T. R. Blaabjerg F, F. Blaabjerg, Z. Chen, R. Teodorescu y F. Lov, «Power Electronics in Wind Turbine Systems,» de Power Electronics and Motion Control Conference, 2006. IPEMC 2006. CES/IEEE 5th International, Shanghai, 2006.

[6] A. Perdana, «Dynamic Models of Wind Turbines,» Ph.D. dissertation, Chalmers Univ. Technol, Gotteborg, Sweden, 2008.

[7] P. M. Achilles S, «Direct Drive Synchronous Machine Models for Stability Assesment of Wind Farms,» de 4th Int. Workshop Large Scale Integration ofWind Power and Transmission Networks for Offshore Windfarms, Billund, Denmark, Oct. 2003.

[8] F. Gonzalez-Longatt, P. Wall y V. Terzija, «A simplified Model for Dynamic Behavior of Permanent Magnet Synchronous Generator for Direct Drive Wind Turbines,» de IEEE Power Tech, Trondheim, 2011.

[9] CDEC-SING, «Anuario y Estadísticas de Operación 2013,» 2013. [En 
línea]. Available: http://www.cdecsing.cl/html docs/anuario2013/idiomas/esp/index.html\#1/z. [Último acceso: $160 \overline{06}$ 2014].

[10] Ministerio de Economía, Fomento y Reconstrucción, «Ley 20.257, Introduce modificaciones a la Ley General de Servicios Eléctricos,» Ministerio de Economía, Santiago, 2008.

[11] Ministerio de Energía, «Ley 20.698, Propicia Ampliación de la Matriz Energética.,» Ministerio de Energía, Santiago, 2013.

[12] Comisión Nacional de Energía, «Norma Técnica de Seguridad y Calidad de Servicio,» Santiago, 2009.

[13] O. Goksu, R. Teodorescu, P. Rodriguez, P. Jansen y L. Helle, «Overwiew of Recent grid Codes for Wind Power Integration,» de 12th International Conference on Optimization of Electrical and Electronic Equipment (OPTIM), 2010.

[14] F. Sada, «Aggregate Model of Large Wind Parks for Power System Studies,» Master's Thesis KTU, Stockholm, Sweden, 2011. 